\title{
Improved sample preparation of beam-sensitive ultra-thin cuprate films
}

Vesna Srot ${ }^{1}$, Yi Wang ${ }^{1}$, Ute Salzberger ${ }^{1}$, Bernhard Fenk $^{1}$, Marion Kelsch ${ }^{1}$, Matteo Minola ${ }^{1}$, Marco Salluzzo $^{2,3}$, Gabriella Maria De Luca ${ }^{3,2}$, Bernhard Keimer ${ }^{1}$ and Peter A. van Aken ${ }^{1}$

${ }^{1 .}$ Max Planck Institute for Solid State Research, Stuttgart, Germany

2. CNR-SPIN Napoli Complesso Monte Sant' Angelo via Cinthia, Napoli, Italy

3. Dipartimento di Fisica “E. Pancini” Complesso Monte Sant' Angelo via Cinthia, Napoli, Italy

* Corresponding author: V.Srot@fkf.mpg.de

Significant progress in technical and methodological development in scanning transmission electron microscopy (STEM) has revealed intriguing phenomena arising at the atomic scale in functional complex oxides. Currently, the quality of electron transparent samples has become one of the major limiting factors for quantitative investigations on the nanoscale.

At present, there is a widespread choice of TEM sample preparation techniques; however, none of them is generally applicable to all materials. During the TEM sample preparation, artifacts may be introduced and therefore special care has to be taken to avoid them. Here, we present two different optimized approaches for achieving high-quality electron transparent TEM lamellae prepared from highly sensitive $\mathrm{NdBa}_{2} \mathrm{Cu}_{3} \mathrm{O}_{7}$ (NBCO) ultra-thin films (4 unit cells thick) that have been deposited on $\mathrm{TiO}_{2}$ terminated $\mathrm{SrTiO}_{3}$ (STO) substrate by high oxygen pressure diode sputtering [1]. Electron transparent lamellae have been investigated by advanced aberration-corrected STEM using a JEOL JEM-ARM200F microscope equipped with a DCOR probe corrector operated at $200 \mathrm{kV}$. STEM images have been obtained using multiple frame acquisition [2] to improve the signal-to-noise ratio as well as to reduce image distortions.

A low-magnification high-angle annular dark-field (HAADF)-STEM image of the NBCO/STO sample in cross-sectional orientation is shown in Figure 1a. The combination of tripod (wedgeshape) polishing followed by $\mathrm{Ar}^{+}$ion-milling, while cooling the sample with $\mathrm{L}-\mathrm{N}_{2}$, was used to prepare the sample. The high-resolution HAADF-STEM image of the NBCO/STO interface shows the high quality sample surface with clearly detectable cation and anion positions (Figure 1b).

In another approach, a focused ion beam (FIB) (Zeiss CrossBeam XB 1540) has been used to prepare the electron transparent TEM lamella in cross-sectional orientation with several electron-transparent regions that are separated by thicker walls (Figure 2a) to reduce the bending of the lamella. Each of the electron transparent areas was thinned by FIB to different thickness ranging from 70 to $150 \mathrm{~nm}$. Afterwards, additional thinning and polishing was done using a Nanomill system (Fischione Model 1040). In such way, we have efficiently removed amorphised material and thinned the sample down to the thickness of $40 \mathrm{~nm}$. The HAADF-STEM image of the NBCO/STO sample shows a clean artefactfree surface (Figure $2 b$ ).

We have shown two different approaches, where the combination of different sample preparation routines are leading to high quality artefact-free samples prepared from highly sensitive ultra-thin cuprate films. With these high quality samples with clean surfaces, further local cation/anion displacement and atomically resolved chemical mapping can be performed [3]. 
References:

[1] M Salluzzo et al. Phys rev B 78 (2008), 054524.

[2] Y Wang et al., Ultramicroscopy 168 (2016) 46.

[3] V Srot et al., Microscopy and Microanalysis 24 (2018) 76.

[4] This project has received funding from the European Union's Horizon 2020 research and innovation programme under grant agreement No. 823717 - ESTEEM3.

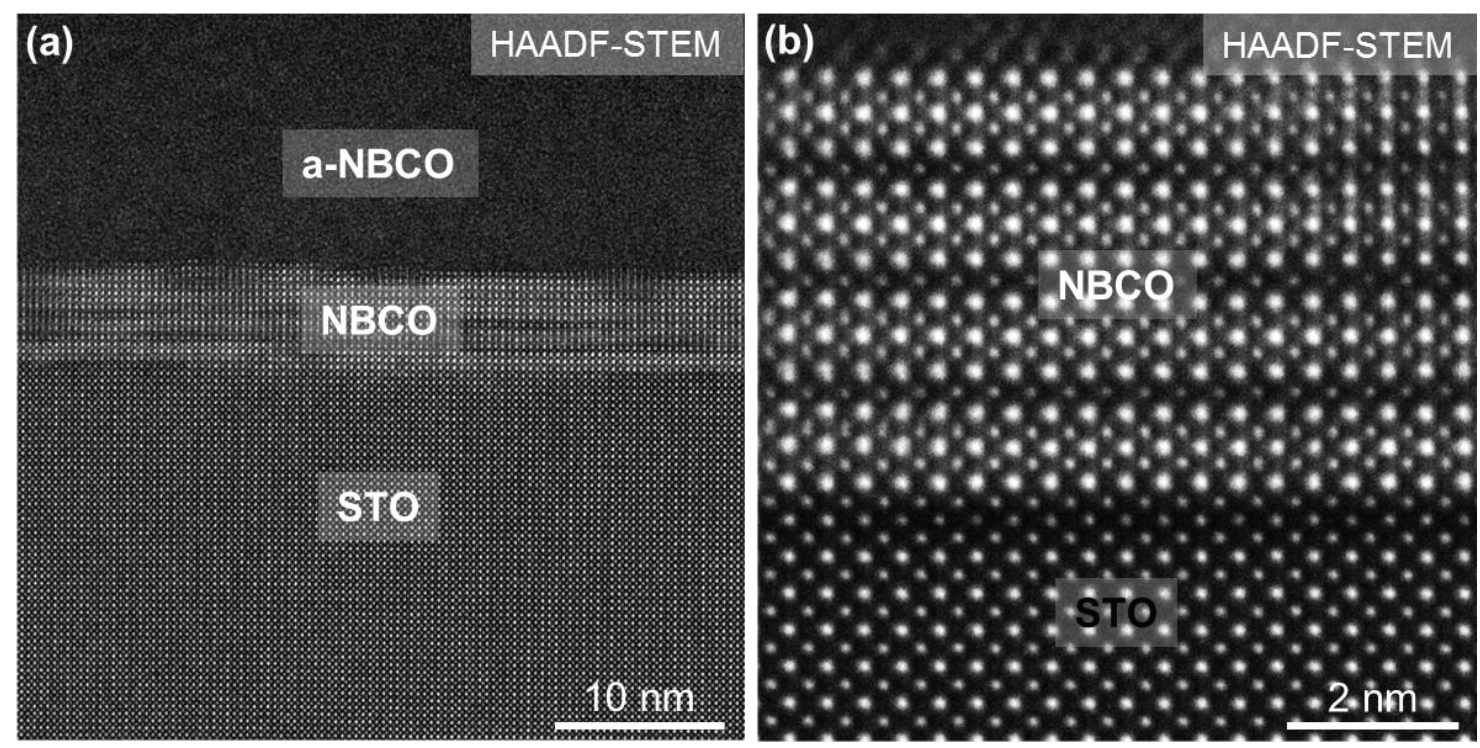

Figure 1. (a) Low magnification HAADF-STEM image of an NBCO ultra-thin film grown on a STO substrate. (b) The atomic arrangement at the NBCO/STO interface has been acquired at higher magnification. The TEM sample has been prepared by a combination of tripod polishing and subsequent $\mathrm{Ar}^{+}$ion-milling while cooling the sample.
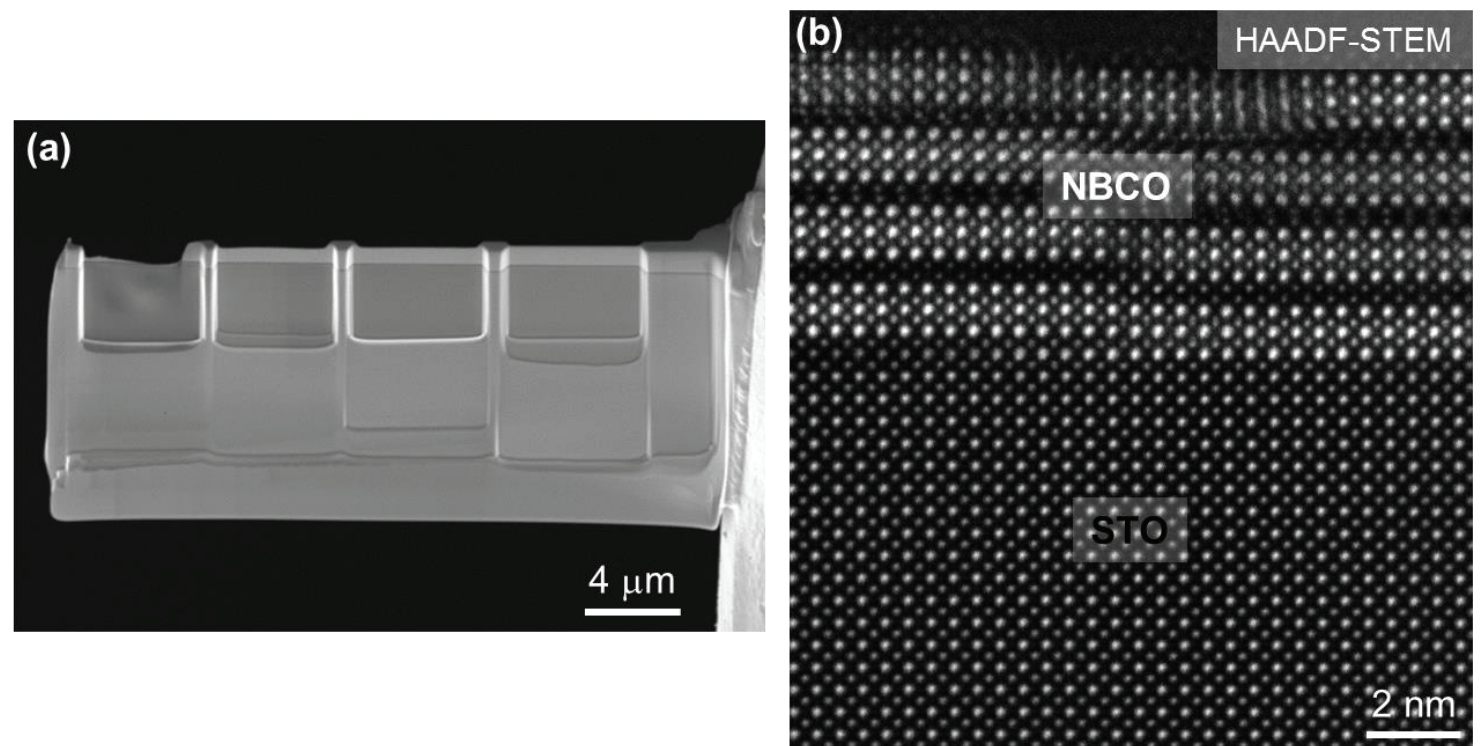

Figure 2. (a) An SEM image of FIB prepared lamellae. (b) HAADF-STEM image of lamellae prepared by FIB that was subsequently thinned and polished by a NanoMill system. Please note that the observed defect is due to a surface step on the STO substrate and is not caused by the TEM sample preparation. 